\title{
The kinetics and mechanisms of hydrolysis and
}

\section{aminolysis of thioxo-cephalosporins}

\author{
Wing Y. Tsang ${ }^{\dagger}$, Anu Dhanda ${ }^{\ddagger}$, Christopher J. Schofield ${ }^{\ddagger}$ and Michael I. Page ${ }^{\dagger *}$ \\ Department of Chemical and Biological Sciences, University of Huddersfield, Queensgate, \\ Huddersfield, HD1 3DH, UK
}

The Dyson Perrins Laboratory and The Oxford Centre for Molecular Sciences, South Parks Road, Oxford, OX1 3QY, UK

m.i.page@hud.ac.uk

RECEIVED DATE (to be automatically inserted after your manuscript is accepted if required according to the journal that you are submitting your paper to)

${ }^{\dagger}$ The University of Huddersfield

†The Dyson Perrins Laboratory 


\section{Experimental details of synthesis and ${ }^{1} \mathrm{H}$ and ${ }^{13} \mathrm{C}$ NMR of (3) and (7) and their precursors.}

\section{Contents}

(6R,7R)-7-(tert-butyloxycarbonyl)amino-3-methyl-8-oxo-5-thia-1-azabicyclo[4,2,0]oct-2-ene-2carboxylic acid $\mathbf{( 1 0})$ page S3

(6R,7R)-diphenylmethyl-7-(tert-butyloxycarbonyl)amino-3-methyl-8-oxo-5-thia-1azabicyclo[4,2,0]oct-2-ene-2-carboxylate (11). page S3

(6R,7R)-diphenylmethyl-7-(tert-butyloxycarbonyl)amino-3-methyl-8-thioxo-5-thia-1azabicyclo[4,2,0]oct-2-ene-2-carboxylate page S4

(6R,7R)-diphenylmethyl-7-amino-3-methyl-8-thioxo-5-thia-1-azabicyclo[4,2,0]oct-2-ene-2carboxylate (13)

\section{S4}

(6R,7R)-diphenylmethyl-7-[

L-(+)-2-(N-tert-butoxycarbonyl)phenylglycine]amino-3-methyl-8thioxo-5-thia-1-azabicyclo[4,2,0]oct-2-ene-2-carboxylate

(14) .page S5

Trifluoroacetic acid salt of (6R,7R)-7-[L-(+)-2-phenylglycine] amino-3-methyl-8-thioxo-5-thia-1azabicyclo[4,2,0]oct-2-ene-2-carboxylic acid (7) page S5

(6R,7R)-diphenylmethyl-7-phenylacetylamino-3-methyl-8-thioxo-5-thia-1-azabicyclo[4,2,0]oct-2ene-2-carboxylate 
$(6 R, 7 R)-7$-phenylacetylamino-3-methyl-8-thioxo-5-thia-1-azabicyclo[4,2,0]oct-2-ene-2-carboxylic

acid

(3)

S6 
(6R,7R)-7-(tert-butyloxycarbonyl)amino-3-methyl-8-oxo-5-thia-1-azabicyclo[4,2,0]oct-2-ene-2-

carboxylic acid (10). To a solution of (6R, 7R)-7-aminodeacetoxycephalosporanic acid (7-ADCA) (6.0 $\mathrm{g}, 28 \mathrm{mmol})$ in saturated aqueous solution of sodium hydrogen carbonate $(300 \mathrm{~mL})$ was added dropwise a solution of di-tert-butyl dicarbonate $97 \%(1.5 \mathrm{eq}, 42 \mathrm{mmol}, 9.45 \mathrm{~g})$ in dioxane $(300 \mathrm{~mL})$. The reaction mixture was stirred at room temperature overnight. Water $(400 \mathrm{~mL})$ was added and the reaction mixture was washed with ethyl acetate $(3 \times 400 \mathrm{~mL})$. The aqueous layer was acidified to $\mathrm{pH} 2$ with $1 \mathrm{~N} \mathrm{HCl}$ and extracted with ethyl acetate $(3 \times 500 \mathrm{~mL})$. The combined organic layers were dried $\left(\mathrm{MgSO}_{4}\right)$, and evaporated under reduced pressure to afford a yellow foam $(6.7 \mathrm{~g}, 76 \%)$, which dried in vacuo. ${ }^{1} \mathrm{H}$ NMR ( $\left.\mathrm{CDCl}_{3}, 200 \mathrm{MHz}\right): 1.47$ (s, 9H), 2.21 (s, 3H), 3.27, 3.57 (ABq, 2H, J=18 Hz), 4.99 (d, 1H, J=4.5 $\mathrm{Hz}, 6 \mathrm{H}), 5.42(\mathrm{~d}, 1 \mathrm{H}, \mathrm{J}=9.5 \mathrm{~Hz}), 5.59(\mathrm{dd}, 1 \mathrm{H}, \mathrm{J}=4.5 \mathrm{~Hz}$ and $\mathrm{J}=9.5 \mathrm{~Hz}, 7 \mathrm{H}), 9.15(\mathrm{~s}, 1 \mathrm{H}) ;{ }^{13} \mathrm{C} \mathrm{NMR}$ (d ${ }^{6}$-DMSO 50.3MHz): 19.8, 28.4, 29.4, 58.2, 61.0, 79.6, 123.6, 131.8, 155.9, 164.4, 165.3. FT-IR

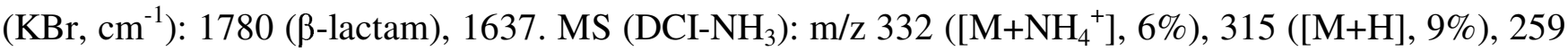
([M-56], 40\%), 187 ([M-157], 80\%), 158 ([M-186], 100\%). Microanalysis: expected C 49.67; H 5.77; N 8.91; S 10.20; found C 49.61; H 5.75; N 8.76; S 10.08.

\section{(6R,7R)-diphenylmethyl-7-(tert-butyloxycarbonyl)amino-3-methyl-8-oxo-5-thia-1-}

azabicyclo[4,2,0]oct-2-ene-2-carboxylate (11). To a solution of cephalosporanic acid (10) (4.65 g, 15 $\mathrm{mmol})$ in dichloromethane $(80 \mathrm{~mL})$ was added dropwise a solution of diphenyldiazomethane in dichloromethane, until a purple solution was obtained. The resultant mixture was stirred for 2 hours. Removal of the solvent under reduced pressure gave a yellow foam, which was purified by column chromatography over silica gel eluting with ethyl acetate:dichloromethane (1:20) to give a white foam (3.60 g, 50\%). ${ }^{1} \mathrm{H}$ NMR (CDCl 3 , 200MHz): 1.47 (s, 9H), 2.16 (s, 3H), 3.22, 3.50 (ABq, 2H, J=18 Hz), $4.96(\mathrm{~d}, 1 \mathrm{H}, \mathrm{J}=4.5 \mathrm{~Hz}, 6 \mathrm{H}), 5.31(\mathrm{~d}, 1 \mathrm{H}, \mathrm{J}=9.5 \mathrm{~Hz}), 5.61(\mathrm{dd}, 1 \mathrm{H}, \mathrm{J}=4.5 \mathrm{~Hz}$ and $\mathrm{J}=9.5 \mathrm{~Hz}, 7 \mathrm{H}), 6.93(\mathrm{~s}$, 1H), 7.31-7.49 (m, 10H); ${ }^{13} \mathrm{C} \mathrm{NMR}\left(\mathrm{CDCl}_{3}, 50.3 \mathrm{MHz}\right): 20.3,28.2,30.3,57.5,60.5,79.0,81.0,122.6$, 127.0, 127.4, 128.0, 128.4, 128.5, 133.1, 139.6, 154.6, 161.4, 165.4. FT-IR (KBr, cm $\left.{ }^{-1}\right): 1780(\beta-$ lactam $),$ 1627. $\mathrm{MS} \quad\left(\mathrm{DCI}-\mathrm{NH}_{3}\right): \quad \mathrm{m} / \mathrm{z} \quad 498 \quad\left(\left[\mathrm{M}+\mathrm{NH}_{4}\right]^{+}, \quad 1.4 \%\right), 167 \quad\left(\left[\mathrm{CH}\left(\mathrm{C}_{6} \mathrm{H}_{5}\right)_{2}\right]^{+}, \quad 100 \%\right)$. Microanalysis: expected C 64.98; H 5.87; N 5.93; S 6.67; found C 65.14; H 5.83; N 5.77; S 6.51. 


\section{(6R,7R)-diphenylmethyl-7-(tert-butyloxycarbonyl)amino-3-methyl-8-thioxo-5-thia-1-}

azabicyclo[4,2,0]oct-2-ene-2-carboxylate (12). To a solution of cephalosporin (11) (3.25 g, 6.76 mmol) in anhydrous toluene $(100 \mathrm{~mL})$ was added Lawesson's reagent $(1.40 \mathrm{~g}, 3.45 \mathrm{mmol})$. The reaction mixture was stirred at $100^{\circ} \mathrm{C}$ overnight ( 20 hours). The solvent was evaporated under reduced pressure. The residue was purified by column chromatography over silica gel eluting with dichloromethane:petroleum ether (40-60) (5:1) to give a golden product $(2.48 \mathrm{~g}, 74 \%) .{ }^{1} \mathrm{H}$ NMR $\left(\mathrm{CDCl}_{3}, 200 \mathrm{MHz}\right): 1.48(\mathrm{~s}, 9 \mathrm{H}), 2.06(\mathrm{~s}, 3 \mathrm{H}), 3.22,3.46(\mathrm{ABq}, 2 \mathrm{H}, \mathrm{J}=18 \mathrm{~Hz}), 5.17$ (d, 1H, J=8.5 Hz), $5.28(\mathrm{dd}, 1 \mathrm{H}, \mathrm{J}=4.0 \mathrm{~Hz}$ and $\mathrm{J}=8.5 \mathrm{~Hz}, 7 \mathrm{H}), 5.42(\mathrm{~d}, 1 \mathrm{H}, \mathrm{J}=4.5 \mathrm{~Hz}, 6 \mathrm{H}), 6.93(\mathrm{~s}, 1 \mathrm{H}), 7.00(\mathrm{~s}, 1 \mathrm{H})$, 7.28-7.40 (m, 10H); ${ }^{13} \mathrm{C} \mathrm{NMR}\left(\mathrm{CDCl}_{3}, 50.3 \mathrm{MHz}\right): 19.9,28.2,30.0,60.3,64.6,78.9,81.0$, 124.6, $127.1,128.0,128.1,128.4,128.4,134.4,139.1,154.8,161.0,201.1$. FT-IR $\left(\mathrm{KBr}, \mathrm{cm}^{-1}\right): 1412(\beta-$ lactam). MS (DCI-NH 3$): \mathrm{m} / \mathrm{z} 498\left(\left[\mathrm{M}+\mathrm{NH}_{4}\right]^{+}, 20 \%\right), 167\left(\left[\mathrm{CH}\left(\mathrm{C}_{6} \mathrm{H}_{5}\right)_{2}\right]^{+}, 100 \%\right)$. Microanalysis: expected C 64.98; H 5.87; N 5.93; S 6.67; found C 65.14; H 5.83; N 5.77; S 6.51.

\section{(6R,7R)-diphenylmethyl-7-amino-3-methyl-8-thioxo-5-thia-1-azabicyclo[4,2,0]oct-2-ene-2-}

carboxylate (13). To a solution of thioxo-cephem (12) (1.18 g, $2.4 \mathrm{mmol})$ in tetrahydrofurandichloromethane $(2: 1, \mathrm{v} / \mathrm{v}, 30 \mathrm{~mL})$ was added toluenesulfonic acid hydrate $(0.46 \mathrm{~g}, 2.4 \mathrm{mmol})$. The reaction mixture was stirred at room temperature for 48 hours. The solvent was evaporated under reduced pressure to dryness and then ethyl acetate $(100 \mathrm{~mL})$ was added. The resultant solution was washed with a saturated aqueous solution of sodium hydrogen carbonate $(2 \times 50 \mathrm{~mL})$, and the organic layer was dried $\left(\mathrm{MgSO}_{4}\right)$ and the solvent evaporated under reduced pressure. The residue was purified by column chromatography over silica gel eluting with ethyl acetate:dichloromethane (5:1) to give a yellow foam (428 mg, 45\%). ${ }^{1} \mathrm{H}$ NMR $\left(\mathrm{CDCl}_{3}, 200 \mathrm{MHz}\right): 1.85$ (s, 2H), 2.02 (s, 3H), 3.23, 3.47 (ABq, 2H, J=18 Hz), $4.43(\mathrm{~d}, 1 \mathrm{H}, \mathrm{J}=4.5 \mathrm{~Hz}, 6 \mathrm{H}), 5.41(\mathrm{~d}, 1 \mathrm{H}, \mathrm{J}=4.5 \mathrm{~Hz}, 7 \mathrm{H}), 7.01(\mathrm{~s}, 1 \mathrm{H}), 7.28-7.40$ (m, $10 \mathrm{H}) ;{ }^{13} \mathrm{C} \mathrm{NMR}\left(\mathrm{CDCl}_{3}, 125.7 \mathrm{MHz}\right): 20.0,29.6,62.76,65.26,78.8,122.0,127.1,127.9,128.1,128.1$ 128.4, 133.4, 139.1, 139.6, 161.0, 207.7. FT-IR (KBr, $\left.\mathrm{cm}^{-1}\right): 1412$ ( $\beta$-lactam). MS (DCI-NH 3$): \mathrm{m} / \mathrm{z} 397$ $([\mathrm{M}+\mathrm{H}], 12 \%), 167\left(\left[\mathrm{CH}\left(\mathrm{C}_{6} \mathrm{H}_{5}\right)_{2}\right]^{+}, 100 \%\right)$. Microanalysis: expected C 63.81; H 5.08; N 7.06; S 16.17; found C 63.78; H 5.07; N 7.10; S 16.09. 


\section{(6R,7R)-diphenylmethyl-7-[ L-(+)-2-(N-tert-butoxycarbonyl)phenylglycine]amino-3-methyl-8-}

thioxo-5-thia-1-azabicyclo[4,2,0]oct-2-ene-2-carboxylate (14). A solution of the free amine (13) (252 $\mathrm{mg}, \quad 0.6 \mathrm{mmol})$ and Boc-protected phenyl glycine (2 eq, $302 \mathrm{mg}, 1.2 \mathrm{mmol})$ in anhydrous tetrahydrofuran $(30 \mathrm{~mL})$ was treated with $\mathrm{EDC}(1.2 \mathrm{eq}, 138 \mathrm{mg}, 0.72 \mathrm{mmol})$ at $0^{\circ} \mathrm{C}$. The resultant mixture was stirred at $0^{\circ} \mathrm{C}$ for 1 hour and at room temperature overnight. The solvent was evaporated under reduced pressure. The residue was purified by column chromatography over silica gel eluting with ethyl acetate:dichloromethane (1:20) to give the thioxo-cephalosporin (7) as a yellow solid (295 mg, 78\%). ${ }^{1} \mathrm{H}$ NMR $\left(\mathrm{CDCl}_{3}, 200 \mathrm{MHz}\right): 1.44$ (s, 9H), 2.05 (s, 3H), 3.19, $3.43(\mathrm{ABq}, 2 \mathrm{H}, \mathrm{J}=18 \mathrm{~Hz}), 5.23$ $(\mathrm{d}, 1 \mathrm{H}, \mathrm{J}=8.5 \mathrm{~Hz}), 5.41(\mathrm{dd}, 1 \mathrm{H}, \mathrm{J}=6.5 \mathrm{~Hz}$ and $\mathrm{J}=4.0 \mathrm{~Hz}, 7 \mathrm{H}), 5.43,5.56(\mathrm{~d}, 1 \mathrm{H}, \mathrm{J}=4.0 \mathrm{~Hz}, 6 \mathrm{H}), 6.45$ (s, 1H), 6.97 (s, 1H), 7.20-7.41 (m, 15H); ${ }^{13} \mathrm{C} \mathrm{NMR}\left(\mathrm{CDCl}_{3}, 50.3 \mathrm{MHz}\right):$ 19.6, 28.4, 31.0, 59.02, 65.4, $65.6,78.4,80.5,125.8,127.8,128.3,128.6,128.9,129.3,129.4,129.6,129.8,136.8,138.8,141.2$, 157.4, 161.8, 172.1, 198.3. FT-IR $\left(\mathrm{KBr}, \mathrm{cm}^{-1}\right): 1385$ ( $\beta$-lactam), 1634. MS (FAB): m/z $630([\mathrm{M}+\mathrm{H}]$, 10\%), $167\left(\left[\mathrm{CH}\left(\mathrm{C}_{6} \mathrm{H}_{5}\right)_{2}\right]^{+}, 100 \%\right)$. Microanalysis: expected C 64.84; H 5.60; N 6.67; S 10.18; found C 64.77; H 5.27; N 6.31; S 10.07.

\section{Trifluoroacetic acid salt of (6R,7R)-7-[L-(+)-2-phenylglycine] amino-3-methyl-8-thioxo-5-thia-} 1-azabicyclo[4,2,0]oct-2-ene-2-carboxylic acid (7). Thioxo-cephem (14) (170 mg, $0.27 \mathrm{mmol})$ was treated with trifluoroacetic acid $(4 \mathrm{~mL})$ and anisole $(0.2 \mathrm{~mL})$ at room temperature overnight. Trifluoroacetic acid was evaporated under reduced pressure to give an oil, which was triturated with anhydrous ether to form a precipitate. Filtration, washing, with cold ether gave a yellow solid (108 mg, 84\%). ${ }^{1} \mathrm{H}$ NMR ((D $\left.{ }_{2} \mathrm{O}, 200 \mathrm{MHz}\right): 2.04$ (s, 3H), 3.35, 3.67 (ABq, 2H, J=18 Hz), 5.07 (1H, m), 5.26, $5.51(\mathrm{dd}, 2 \mathrm{H}, \mathrm{J}=4 \mathrm{~Hz}, 6 \mathrm{H}, 7 \mathrm{H}), 7.31(\mathrm{~m}, 5 \mathrm{H}) . \mathrm{FT}-\mathrm{IR}\left(\mathrm{KBr}, \mathrm{cm}^{-1}\right): 1676,1410(\beta-\mathrm{lactam}) . \mathrm{MS}(\mathrm{ESI}): \mathrm{m} / \mathrm{z}$ $364(\mathrm{M}+\mathrm{H}, 100 \%)$.

\section{(6R,7R)-diphenylmethyl-7-phenylacetylamino-3-methyl-8-thioxo-5-thia-1-azabicyclo[4,2,0]oct-}

2-ene-2-carboxylate (15). Phenylacetyl chloride (1.1 eq , $0.58 \mathrm{mmol}, 83 \mu \mathrm{L})$ was added dropwise to a solution of amine $(13)(210 \mathrm{mg}, 0.53 \mathrm{mmol})$ in tetrahydrofuran $(20 \mathrm{~mL})$ at $0^{\circ} \mathrm{C}$. The reaction mixture 
was stirred at $0^{\circ} \mathrm{C}$ for 3 hours. The solvent was removed under reduced pressure and the residue was purified by column chromatography over silica gel eluting with ethyl acetate:petroleum ether (40-60) (1:1) to yield a colourless oil $(270 \mathrm{mg}, 99 \%) .{ }^{1} \mathrm{H}$ NMR $\left(\mathrm{CDCl}_{3}, 400 \mathrm{MHz}\right): 2.00$ (s, 3H), 3.10, 3.40 $(\mathrm{ABq}, 2 \mathrm{H}, \mathrm{J}=18 \mathrm{~Hz}), 3.66(\mathrm{~s}, 2 \mathrm{H}, \mathrm{J}=8 \mathrm{~Hz}), 5.38(\mathrm{~d}, 1 \mathrm{H}, \mathrm{J}=4 \mathrm{~Hz}), 5.46(\mathrm{dd}, 2 \mathrm{H}, \mathrm{J}=4 \mathrm{~Hz}, 6 \mathrm{H}, 7 \mathrm{H}), 6.02$ $(1 \mathrm{H}, \mathrm{d}, \mathrm{J}=8 \mathrm{~Hz}), 7.30(\mathrm{~m}, 5 \mathrm{H}) ;{ }^{13} \mathrm{C} \mathrm{NMR}\left(\mathrm{CDCl}_{3}, 400 \mathrm{MHz}\right) 20.0,35.0,41.4,59.3,69.0,79.3,125$, 127.5, 127.9, 128.6, 129.3 and 129.6, 133.9, 133.8, 172.1, 176.1, 200.0. ESI 515.1465 (calculated for $\mathrm{C}_{29} \mathrm{H}_{27} \mathrm{~N}_{2} \mathrm{O}_{3} \mathrm{~S}_{2}$ 515.1463).

\section{$(6 R, 7 R)-7-p h e n y l a c e t y l a m i n o-3-m e t h y l-8-t h i o x o-5-t h i a-1-a z a b i c y c l o[4,2,0]$ oct-2-ene-2-}

carboxylic acid (3). Thioxo-cephalosporin (15) (230 mg, $0.45 \mathrm{mmol}$ ) was treated with trifluoroacetic acid $(3 \mathrm{~mL})$ in the presence of anisole $(0.15 \mathrm{~mL})$ at room temperature overnight. Trifluoroacetic acid was evaporated under reduced pressure to give an oil, which was triturated with anhydrous ether to form a precipitate. Filtration, washing, with cold ether gave a colourless oil (166 mg, 80\%). ${ }^{1} \mathrm{H}$ NMR $\left(\left(\mathrm{CD}_{3}\right)_{2} \mathrm{SO}, 500 \mathrm{MHz}\right): 2.02(\mathrm{~s}, 3 \mathrm{H}), 3.45,3.65(\mathrm{ABq}, 2 \mathrm{H}, J=18 \mathrm{~Hz}), 3.57(\mathrm{t}, 2 \mathrm{H}, J=8 \mathrm{~Hz}), 5.39$ (dd, 2H, $J=4 \mathrm{~Hz}), 5.54(\mathrm{~d}, 1 \mathrm{H}, J=4 \mathrm{~Hz}), 7.30(\mathrm{~m}, 5 \mathrm{H}), 8.8(\mathrm{~d}, 1 \mathrm{H}, J=8.5 \mathrm{~Hz}) ;{ }^{13} \mathrm{C} \mathrm{NMR}\left(\left(\mathrm{CD}_{3}\right)_{2} \mathrm{SO}, 500 \mathrm{MHz}\right)$ : $20.0,30.0,41.4,59.3,65.0,125,125.7,127.1,128.6,130.0,133.6,135.7,172.1,173.2,200.0$. ESI 349.0697 (calculated for $\mathrm{C}_{16} \mathrm{H}_{17} \mathrm{~N}_{2} \mathrm{O}_{3} \mathrm{~S}_{2} 349.0681$ ). 